One interpretation of these two studies is that miR-17-92 miRNAs are oncogenic but can also have tumor-suppressor effects by decreasing E2F1 translation ${ }^{16}$. This is probably an oversimplification, as E2F1 has been shown to function both as oncogene and tumor suppressor ${ }^{17}$. In line with this notion, decreased E2F1 translation in the miR-17-92 mouse lymphoma could explain the reduced apoptosis. It is now of great interest to analyze in detail human lymphomas with $m i R-17-92$ overexpression.

The data from these studies suggest a simple framework to understand the role of miRNAs in cancer: miRNA-mediated tumorigenesis results from either downregulation of tumor suppressor genes or upregulation of oncogenes (Fig. 1).

Lu et al. ${ }^{3}$ asked whether miRNA expression patterns could be used to classify human cancer. To do this reliably, they first developed a sensitive bead-based hybridization technology with higher specificity than microarray methods. They then measured the expression of all 217 known human miRNAs in a panel of 218 human cancer samples.

Hierarchical cluster analysis showed that cancers from similar developmental origins grouped together, with clear partitioning of hematopoietic from epithelial cancers. Gastrointestinal cancers, which arise from endoderm, were also clustered together. In contrast to the miRNA results, data from mRNA microarray profiling did not provide accurate classification.

Stratification within cancer types using miRNA expression was also achieved; as comparison between subtypes of acute lymphoblastic leukemia samples also showed clear partitioning into previously characterized molecular classifications (BCR/ABL, Tcell acute lymphoblastic leukemia and mixed lineage leukemia). Strikingly, cancers in both human and mouse had lower expression of a large proportion of miRNAs as compared to normal tissues. Moreover, using two different cellular systems, they showed upregulation of miRNAs as cells differentiated. This led the authors to suggest that lower levels of miRNAs reflect loss of differentiation, which is a cardinal feature of cancer.

These three studies establish the miRNAcancer connection: two give us mechanistic insights about how misexpression of miRNA might disrupt tumorigenic networks and the third shows the promise of using miRNA profiling for cancer classification.

The miRNA expression profiling report has profound clinical implications if expression levels of 200 miRNAs can reproducibly classify cancers. The stability of miRNAs and the ability to isolate them intact (even from paraffin-embedded tissues) will prompt many studies to show the prognostic and predictive value of miRNA profiling, given that there are now several high-throughput platforms to do so. Time will tell whether miRNA profiling will supersede mRNA profiling.

An even more exciting prospect is the potential for analysis of patterns of tissue miRNA expression using in situ hybridization. This is now possible using special high-affinity RNA analog probes ${ }^{18}$ and would facilitate tissue- level studies of preneoplastic lesions, tumor microenvironment, clonal heterogeneity, invasion and metastasis.

As key regulators of gene expression, miRNAs may also have a role in genetic predisposition to disease. The recent description of a sequence polymorphism in a mature miRNA ${ }^{19}$ raises the prospect of testing the association of such polymorphisms with cancer. Therapeutic targeting of these tiny RNA molecules is attractive and, in theory, technically possible, using miRNA silencing induced by small interfering RNA ${ }^{5,6}$. Such approaches will have to wait for further understanding of regulation of oncogenic and tumor suppressive networks by miRNAs before their use in the clinic can be contemplated.

1. He, L. et al. Nature 435, 828-833 (2005).

2. O'Donnell K. A. et al. Nature 435, 839-843 (2005).

3. Lu, J. et al. Nature 435, 834-838 (2005).

4. Bartel, D. P. Cell 116, 281-297 (2004).

5. He, L. et al. Nat. Rev. Genet. 5, 522-531 (2004)

6. Ambros, V. Nature 431, 350-355 (2004)

7. Lee, R. C. et al. Cell 75, 843-854 (1993).

8. Reinhart, B. J. et al. Nature 403, 901-906 (2000).

9. Berezikov, E. et al. Cell 120, 21-24 (2005).

10. Lewis, B. P. et al. Cell 120, 15-20 (2005).

11. Krek, A. et al. Nat. Genet. 37, 495-500 (2005).

12. Calin, G. A. et al. Proc. Natl. Acad. Sci. USA 99 15524-15529 (2002).

13. Michael, M. Z. et al. Mol. Cancer Res. 1, 882-891 (2003).

14. Johnson, S. M. et al. Cell 120, 635-647 (2005).

15. Dimova, D. K. et al. Oncogene 24, 2810-2826 (2005).

16. Meltzer, P. S. Nature 435, 745-746 (2005)

17. Weinberg, R. A. Cel/ 85, 457-459 (1996).

18. Weinholds, E. et al. Science published online 26 May 2005 (doi:10.1126/science.1114519).

19. Iwai, N. et al. Biochem. Biophys. Res. Commun. 331, 1439-1444 (2005)

\title{
microRNAs manage the heart
}

Genes encoding microRNAs-already thought to be involved in the control of developmental events-have now found their way into the heart, according to a study in a recent issue of Nature (advanced online publication 12 June 2005; doi:10.1038/nature03817).

Yong Zhao et al. focused on two related microRNAs expressed in the heart. Shown is the expression pattern of one of these microRNAs, miR-1-1, in the mouse embryo. miR-1-1 is found in the developing heart (dark blue) and in the somites, embryonic structures that give rise to muscle.

The researchers next devised a new algorithm to search for microRNA target sequences in mRNA. Both microRNAs, they found, negatively regulate Hand2, a transcription factor that promotes expansion of ventricular heart muscle precursor cells. In line with that finding, overexpression of the microRNAs in the heart decreased the pool of proliferating precursor cells.

The authors also identified transcriptional regulators that control expression of the microRNAs. These included MyoD and other regulators of muscle differentiation. The data suggest that these heart microRNAs control the balance between differentiation and proliferation during heart development.

\section{Charlotte Schubert}

